Supporting Information for

\title{
Development of predictive models for "very poor" beach water quality gradings using class-imbalance learning
}

\author{
Jiuhao GUO ${ }^{1}$, Joseph H. W. LEE ${ }^{2,3}$ \\ ${ }^{1}$ Department of Civil and Environmental Engineering, The Hong Kong University of Science and \\ Technology, Clear Water Bay, Hong Kong, China \\ ${ }^{2}$ Macau Environmental Research Institute, Macau University of Science and Technology, Taipa, \\ Macao, China \\ ${ }^{3}$ Institute for Advanced Study, The Hong Kong University of Science and Technology, Clear Water \\ Bay, Hong Kong, China
}

Contents -6 pages

- Table S1: Summary of variables and sources of data used in this study

- A description on illustrating the growing of a decision tree stump using 1990's data at Big Wave Bay

- Table S2: Training data in year 1990 at Big Wave Bay for illustration of decision stump growing.

- Figure S1: Computation table showing the determination of best split for each feature in decision stump growing.

- Figure S2: Summary of best split point for each feature (upper table) and the final decision tree. 
Table S1: Summary of variables and sources of data used in this study

\begin{tabular}{|c|c|c|c|}
\hline \multirow{2}{*}{ Abbreviation } & \multirow{2}{*}{ Variable (unit) } & \multicolumn{2}{|l|}{ Source } \\
\hline & & Regular data* & Daily data $^{* *}$ \\
\hline$\overline{\mathbf{E C}}$ & "Measured E. coli concentration (counts/100mL) & \multirow{2}{*}{$\begin{array}{l}\mathrm{EPD}^{\wedge} \\
\text { (https://cd.epic.epd.gov.hk/EPICDI/beach/requestinfo/) }\end{array}$} & \multirow{4}{*}{$\begin{array}{l}\text { Thoe } 2010^{\#} \\
\text { (available upon request) }\end{array}$} \\
\hline EC5 & $\begin{array}{l}\text { Geometric mean of } E \text {. coli concentration in the } \\
\text { past five water samples (counts } / 100 \mathrm{~mL} \text { ) }\end{array}$ & & \\
\hline Temp. & In-situ measured water temperature $\left({ }^{\circ} \mathrm{C}\right)$ & $\mathrm{HKO}^{\wedge \wedge}$ (https://www.hko.gov.hk/en/cis/climat.htm) & \\
\hline Sal. & In-situ measured water salinity (psu) & EPD (available upon request) & \\
\hline Tide & $\begin{array}{l}\text { Tide level around 10:00 am at the nearest tidal } \\
\text { gauge station }(\mathrm{m})\end{array}$ & \multicolumn{2}{|c|}{ Predicted by Extended Harmonic Analysis, tidal constituents obtained from HKO } \\
\hline 1d-Rain & $\begin{array}{l}\text { Daily rainfall at the nearest weather station in } \\
\text { one day earlier }(\mathrm{mm})\end{array}$ & \multirow{5}{*}{\multicolumn{2}{|c|}{ HKO (https://www.hko.gov.hk/en/cis/climat.htm) }} \\
\hline 2d-Rain & $\begin{array}{l}\text { Daily rainfall at the nearest weather station in } \\
\text { two days earlier }(\mathrm{mm})\end{array}$ & & \\
\hline 3d-Rain & $\begin{array}{l}\text { Daily rainfall at the nearest weather station in } \\
\text { three days earlier }(\mathrm{mm})\end{array}$ & & \\
\hline Radiation & $\begin{array}{l}\text { Cumulative solar radiation at King's Park in the } \\
\text { previous day }\left(\mathrm{MJ} / \mathrm{m}^{2}\right)\end{array}$ & & \\
\hline Wind & $\begin{array}{l}\text { Prevailing on-shore wind speed at Waglan } \\
\text { Station in the previous day }(\mathrm{m} / \mathrm{s})\end{array}$ & & \\
\hline
\end{tabular}

$\wedge \quad$ Environmental Protection Department, Government of the Hong Kong Special Administrative Region of China

$\wedge \wedge \quad$ Hong Kong Observatory, Government of the Hong Kong Special Administrative Region of China

* Sampling interval: 3 to 14 days; time coverage: 1990-2020 for all beaches;

** Sampling interval: daily; time coverage: 1 June to 31 July 2007 for Big Wave Bay, 14 July to 13 October 2008 for both Big Wave Bay and Silvermine Bay;

\# Thoe, W. A Daily Forecasting System of Marine Beach Water Quality in Hong Kong. Ph.D. thesis, The University of Hong Kong, HKSAR, China, 2010. 


\section{Illustration of growing decision tree stump using 1990's data at Big Wave Bay}

As an example for better illustration of the decision stump (one split from root node to two leaf nodes) growing process, we demonstrate here an example using the data of Big Wave Bay in year 1990 only for training. Table S1 shows a summary of the training data. Each feature vector has 9 features or independent variables. Each row represents the date of the observation, the observed values of the feature vector, the observed E.coli concentration (EC), and the associated class label. There are a total of 34 data points, with 6 points belonging to positive class $(>610)$ and 28 points to negative $(\leq 610)$.

The training, or growing, process can be illustrated by a computation chart as shown in Fig. S1. As the first step, the algorithm first sort the rows of observations (or examples) by ascending one of the features (e.g. EC5). Then a set potential split points are calculated by averaging the feature values for every two adjacent observations. For each potential split point, the algorithm classifies an observation as positive when EC5 $\leq$ split value and negative when otherwise. Gini impurities for positive and negative prediction $(I G+$ and $I G-)$, together with that for the stump (\$IG\$) are then computed for each potential split value using Eq. 1, 2 and 3 in the main text. Then a best split for this test feature will be selected by finding the split value with minimum values of $I G$.

The above process is repeated for all input features by the algorithm. Using the AdaBoost method the final decision tree (stump) will be based on only one feature ("weak learner") - the feature with the smallest value of minimum \$IG\$. As shown in Fig. S2, in this example the condition of "If salinity $\leq 22.25$ then positive" gives the smallest value of minimum $I G$ and hence a stump using this condition is selected. However, the final classifier is derived from an ensemble of "weak learners" (Eq.8). 
Table S2: Training data in year 1990 at Big Wave Bay for illustration of decision stump growing.

\begin{tabular}{|c|c|c|c|c|c|c|c|c|c|c|c|}
\hline \multirow[b]{2}{*}{ Date } & \multicolumn{9}{|c|}{ Features } & \multirow[b]{2}{*}{$\begin{array}{c}\text { EC } \\
\text { counts } / 100 \mathrm{~mL}\end{array}$} & \multirow[b]{2}{*}{ Label } \\
\hline & $\begin{array}{c}\text { EC5 } \\
\text { counts } / 100 \mathrm{~mL}\end{array}$ & $\begin{array}{c}\text { 1d-Rain } \\
\mathrm{mm}\end{array}$ & $\begin{array}{c}\text { 2d-Rain } \\
\mathrm{mm}\end{array}$ & $\begin{array}{c}\text { 3d-Rain } \\
\text { mm }\end{array}$ & $\begin{array}{c}\text { Radiation } \\
\mathrm{MJ} / \mathrm{m} 2\end{array}$ & $\begin{array}{c}\text { Tide } \\
\text { m }\end{array}$ & $\begin{array}{c}\text { Temp. } \\
\text { oc }\end{array}$ & $\begin{array}{l}\text { Sal. } \\
\text { psu }\end{array}$ & $\begin{array}{l}\text { Wind } \\
\mathrm{m} / \mathrm{s}\end{array}$ & & \\
\hline 3-Jan-90 & 37.6 & 1.0 & 1.0 & 16.5 & 5.49 & 1.19 & 17.7 & 25.0 & -3.68 & 78 & $<=610$ \\
\hline 17-Jan-90 & 33.5 & 0.0 & 3.3 & 0.0 & 7.45 & 1.30 & 17.0 & 26.1 & -3.76 & 122 & $<=610$ \\
\hline 6-Feb-90 & 56.5 & 0.0 & 0.0 & 0.0 & 10.92 & 1.36 & 16.0 & 25.9 & 0.00 & 180 & $<=610$ \\
\hline 20-Feb-90 & 139.0 & 0.0 & 7.0 & 26.5 & 13.60 & 1.49 & 16.9 & 27.0 & 0.81 & 280 & $<=610$ \\
\hline 7-Mar-90 & 128.1 & 0.0 & 0.0 & 0.5 & 17.76 & 1.48 & 16.9 & 28.0 & -3.75 & 30 & $<=610$ \\
\hline 14-Mar-90 & 107.5 & 0.0 & 0.0 & 58.0 & 13.07 & 1.93 & 16.8 & 27.0 & 5.31 & 200 & $<=610$ \\
\hline 21-Mar-90 & 129.8 & 0.0 & 0.0 & 0.0 & 22.11 & 1.69 & 18.3 & 22.5 & 1.21 & 3 & $<=610$ \\
\hline 5-Apr-90 & 61.9 & 25.0 & 0.0 & 0.0 & 3.96 & 1.54 & 19.5 & 24.0 & -5.46 & 160 & $<=610$ \\
\hline $11-A p r-90$ & 60.4 & 8.5 & 37.0 & 0.0 & 16.16 & 2.05 & 20.3 & 22.0 & 1.66 & 5900 & $>610$ \\
\hline 19-Apr-90 & 111.2 & 1.0 & 3.0 & 4.0 & 6.36 & 1.76 & 19.9 & 25.0 & 5.90 & 300 & $<=610$ \\
\hline 3-May-90 & 176.2 & 0.0 & 0.0 & 0.0 & 24.29 & 1.61 & 23.3 & 28.0 & 0.85 & 15 & $<=610$ \\
\hline 9-May-90 & 105.0 & 0.0 & 0.0 & 0.0 & 17.71 & 2.02 & 22.2 & 20.0 & 2.82 & 33000 & $>610$ \\
\hline 16-May-90 & 675.1 & 0.0 & 0.0 & 12.0 & 9.75 & 2.12 & 23.3 & 25.0 & 9.04 & 140 & $<=610$ \\
\hline 23-May-90 & 657.3 & 0.0 & 0.0 & 0.0 & 15.04 & 1.65 & 24.1 & 24.0 & 3.40 & 140 & $<=610$ \\
\hline 6-Jun-90 & 311.0 & 2.5 & 0.0 & 2.0 & 10.63 & 1.71 & 25.3 & 21.0 & 3.75 & 1000 & $>610$ \\
\hline 13-Jun-90 & 395.7 & 0.0 & 0.5 & 3.0 & 20.14 & 2.12 & 28.3 & 24.0 & 1.89 & 330 & $<=610$ \\
\hline 21-Jun-90 & 734.3 & 0.0 & 1.1 & 11.2 & 19.03 & 1.70 & 29.0 & 24.0 & 4.34 & 90 & $<=610$ \\
\hline 4-Jul-90 & 225.4 & 2.3 & 0.0 & 0.1 & 17.01 & 1.34 & 29.1 & 19.0 & 4.39 & 290 & $<=610$ \\
\hline 11-Jul-90 & 260.8 & 0.0 & 0.0 & 0.0 & 24.87 & 2.12 & 27.6 & 25.0 & -0.95 & 150 & $<=610$ \\
\hline 18-Jul-90 & 264.4 & 4.0 & 0.0 & 1.5 & 22.47 & 1.06 & 27.2 & 23.0 & 0.00 & 400 & $<=610$ \\
\hline 1-Aug-90 & 220.1 & 70.0 & 23.5 & 0.5 & 5.30 & 1.14 & 27.5 & 24.0 & -8.42 & 50 & $<=610$ \\
\hline 8-Aug-90 & 150.9 & 0.0 & 1.0 & 7.0 & 25.16 & 2.15 & 27.2 & 26.0 & 0.00 & 1 & $<=610$ \\
\hline 15 -Aug-90 & 61.4 & 0.0 & 0.0 & 0.0 & 14.85 & 0.90 & 28.7 & 26.0 & 2.32 & 9 & $<=610$ \\
\hline 22-Aug-90 & 30.6 & 0.0 & 0.0 & 0.0 & 7.24 & 2.13 & 24.7 & 29.0 & -6.64 & 60 & $<=610$ \\
\hline 5-Sep-90 & 25.5 & 0.0 & 0.0 & 0.0 & 18.95 & 2.02 & 29.0 & 26.0 & 4.55 & 140 & $<=610$ \\
\hline $11-$ Sep-90 & 20.7 & 68.0 & 7.0 & 25.0 & 1.36 & 1.08 & 26.3 & 22.0 & -6.62 & 1100 & $>610$ \\
\hline 20-Sep-90 & 38.4 & 55.0 & 0.0 & 0.0 & 2.17 & 1.97 & 28.1 & 23.0 & 8.75 & 690 & $>610$ \\
\hline 3-Oct-90 & 141.8 & 0.0 & 0.0 & 0.0 & 11.35 & 1.88 & 26.8 & 25.0 & 8.66 & 33 & $<=610$ \\
\hline $10-$ Oct-90 & 183.9 & 0.5 & 0.0 & 0.0 & 20.26 & 0.94 & 27.0 & 25.0 & 6.79 & 52 & $<=610$ \\
\hline $17-$ Oct-90 & 178.7 & 0.0 & 0.0 & 0.0 & 18.72 & 1.65 & 26.2 & 25.0 & 7.91 & 180 & $<=610$ \\
\hline 8-Nov-90 & 187.9 & 0.0 & 0.0 & 0.0 & 10.14 & 0.85 & 24.0 & 24.0 & 8.64 & 70 & $<=610$ \\
\hline 22-Nov-90 & 108.3 & 0.0 & 0.0 & 0.0 & 6.78 & 1.06 & 22.4 & 24.0 & -3.54 & 1300 & $>610$ \\
\hline 6-Dec-90 & 123.0 & 0.0 & 0.0 & 0.0 & 14.94 & 0.98 & 20.5 & 26.0 & -2.94 & 72 & $<=610$ \\
\hline 20-Dec-90 & 143.7 & 0.0 & 0.0 & 0.0 & 11.00 & 1.12 & 20.3 & 31.0 & 6.43 & 220 & $<=610$ \\
\hline
\end{tabular}

- $\quad$ EC: measured E. coli concentration

- EC5: geometric mean of the past 5 most recent measurements of $E$. coli concentrations

- 1d-, 2d-, and 3d-Rain: total daily rainfall 1-day, 2-day, and 3-day prior to the sampling day

- Radiation: previous day's solar radiation

- Tide: predicted tidal level at the typical time when water samples are collected (around 10:00 am)

- Temp.: in-situ measured water temperature

- Sal.: in-situ measured salinity

- Wind: yesterday's prevailing onshore wind speed based on wind speed and direction data at Waglan Island 
Step 1:

Sort the examples by ascending the test feature (e.g. EC5)

\begin{tabular}{|c|c|c|c|c|c|c|c|c|c|c|}
\hline \multirow{2}{*}{ Date } & \multirow{2}{*}{ Label } & \multirow[b]{2}{*}{ EC5 } & \multirow{2}{*}{ Split } & \multicolumn{3}{|c|}{$\begin{array}{c}>610 \text { (Positive) } \\
\text { Criterion: EC5<=split }\end{array}$} & \multicolumn{3}{|c|}{$\begin{array}{l}<=610 \text { (Negative) } \\
\text { Criterion: EC5>split }\end{array}$} & \multirow{2}{*}{ IG } \\
\hline & & & & $\begin{array}{l}\text { Correct } \\
(\mathrm{NCj}+)\end{array}$ & $\begin{array}{l}\text { Wrong } \\
\text { (NCj-) }\end{array}$ & IG+ & $\begin{array}{l}\text { Correct } \\
(\mathrm{NCj}+)\end{array}$ & $\begin{array}{l}\text { Wrong } \\
\text { (NCj-) }\end{array}$ & IG- & \\
\hline $11-$-ер-1990 & 2610 & 20.7 & i & & & & & & & \\
\hline 05-Sep-1990 & $<=610$ & 25.5 & 23.1 & 1 & 0 & 0.000 & 28 & 5 & 0.257 & 0.250 \\
\hline 22-Aug-1990 & $<=610$ & 30.6 & 28.1 & 1 & 1 & 0.500 & 27 & 5 & 0.264 & 0.278 \\
\hline $17-\operatorname{Jan}-1990$ & $<=610$ & 33.5 i & 32.1 & 1 & 2 & 0.444 & 26 & 5 & 0.271 & 0.286 \\
\hline 03-Jan-1990 & $<-610$ & 37.6 & 35.6 & 1 & 3 & 0.375 & 25 & s & 0.278 & 0.289 \\
\hline 20-Sep-1990 & $\Varangle 610$ & 38.4 i & 380 i & 1 & 4 & 0.320 & 24 & 5 & 0.285 & 0.290 \\
\hline 06-Feb-1990 & $<=610$ & 56.5 & 47.4 & 12 & 4 & 0.444 & 24 & 4 & 0.245 & 0.280 \\
\hline $11-A p r-1990$ & $\times 610$ & i 60.4 i & ; 58.5 & 2 & 5 & 0.408 & 23 & 4 & 0.252 & 0.284 \\
\hline 15-Aug-1990 & $<=610$ & 61.4 & 60.9 ; & 13 & 5 & 0.469 & 23 & 3 & 0.204 & 0.266 \\
\hline OS-Apr-1990 & $<-610$ & 61.9 & 61.6 & 3 & 6 & 0.444 & 22 & 3 & 0.211 & 0.273 \\
\hline 09-May-1990 & $>610$ & $105.0\}$ & ; 83.4 i & 3 & 7 & 0.420 & 21 & 3 & 0.219 & 0.278 \\
\hline $14-\mathrm{Mar}-1990$ & $<-610$ & 107.5 & 106.3 & 4 & 7 & 0.463 & 21 & 2 & 0.159 & 0.257 \\
\hline 22-Nov-1990 & $\times 610$ & 108.3 & 107.9 & 4 & 8 & 0.444 & 20 & 2 & 0.165 & 0.264 \\
\hline 19-Apr-1990 & $<-610$ & 111.2 & $7 \overline{109.8-1}$ & $7-$ & $\overline{8}$ & $\overline{0.4 \overline{7} 3}$ & $2 \overline{0}$ & $\overline{1}$ & $\overline{0.09} \overline{1}$ & $\overline{0.237} ;$ \\
\hline 06-Dec-1990 & $<=610$ & 123.0 & :117า? & $r-5$ & $9^{-}$ & 0.459 & 19 & $\mathrm{~T}^{-}$ & 0.095 & $6.245^{-}$ \\
\hline 07-Mar-1990 & $<=610$ & | 128.1$\}$ & 125.5 & 5 & 10 & 0.444 & 18 & 1 & 0.100 & 0.252 \\
\hline $21-\mathrm{Mar}-1990$ & $<=610$ & 129.8 & 129.0 & 5 & 11 & 0.430 & 17 & 1 & 0.105 & 0.258 \\
\hline $20-F e b-1990$ & $c-610$ & 139.01 & 134.4 & 5 & 12 & 0.415 & 16 & 1 & 0.111 & 0.263 \\
\hline $03-0 x t-1990$ & $<-610$ & 141.8 ' & ! & 5 & 13 & 0.401 & 15 & 1 & 0.117 & 0.268 \\
\hline 20-Dec-1990 & $c-610$ & 143.7 & 142.8 ! & 15 & 14 & 0.388 & 14 & 1 & 0.124 & 0.272 \\
\hline 08-Aug-1990 & $<=610$ & $150.9 !$ & ist.3 & 5 & 15 & 0.375 & 13 & 1 & 0.133 & 0.275 \\
\hline 03-May-1990 & $<=610$ & 176.2 & 163.6 ! & 15 & 16 & 0.363 & 12 & 1 & 0.142 & 0.278 \\
\hline $17-$ Oct-1990 & $<=610$ & I 178.7 & i177.5 & 5 & 17 & 0.351 & 11 & 1 & 0.153 & 0.281 \\
\hline $10-0 c t-1990$ & $c-610$ & $183.9 !$ & 181.3 & 5 & 18 & 0.340 & 10 & 1 & 0.165 & 0.284 \\
\hline 08-Nov-1990 & $<-610$ & 187.9 & 185.9 i & 5 & 19 & 0.330 & 9 & 1 & 0.180 & 0.286 \\
\hline 01 -Aug- 1990 & $<=610$ & 220.11 & 204.0 & 5 & 20 & 0.320 & 8 & 1 & 0.198 & 0.288 \\
\hline 04-Jul-1990 & $<=610$ & 225.4 & '222.8 ! & 5 & 21 & 0.311 & 7 & 1 & 0.219 & 0.289 \\
\hline 11-Jul-1990 & $<=610$ & 260.8 & i243.1 i & 5 & 22 & 0.302 & 6 & 1 & 0.245 & 0.290 \\
\hline 18-Jul-1990 & $<-610$ & ' 264.4 ' & '262.6 & 5 & 23 & 0.293 & 5 & 1 & 0.278 & 0.291 \\
\hline 06-Jun-1990 & $\$ 610$ & 311.0 & 287.7 & 5 & 24 & 0.285 & 4 & 1 & 0.320 & 0.290 \\
\hline 13-Jun-1990 & $<-610$ & i 395.7 i & 353.4 & 6 & 24 & 0.320 & 4 & 0 & 0.000 & 0.282 \\
\hline 23-May-1990 & $<=610$ & $657.3\}$ & ?526.5 ! & 16 & 25 & 0.312 & 3 & 0 & 0.000 & 0.285 \\
\hline 16-May-1990 & $<=610$ & 675.1 & 1666.2 & 6 & 26 & 0.305 & 2 & 0 & 0.000 & 0.287 \\
\hline 21-Jun-1990 & $<-610$ & $734.3 i$ & 704.7 & 6 & 27 & 0.298 & 1 & 0 & 0.000 & 0.289 \\
\hline
\end{tabular}

Step 3:

Classify examples based on each test criterion and calculate the corresponding Gini impurity (IG+, IG- and IG as computed by Eq. 1, 2 and 3)
Step 4:

Select best split with minimum IG

Figure S1: Computation table showing the determination of best split for each feature in decision stump growing. 


\begin{tabular}{cccccccccc}
\hline & EC5 & 1d-Rain & 2d-Rain & 3d-Rain & Radiation & Tide & Temp. & Sal. & Wind \\
\hline min. IG & 0.237 & 0.210 & 0.250 & 0.289 & 0.206 & 0.269 & 0.268 & $\underline{0.157}$ & 0.278 \\
Best split & 109.8 & 2.40 & 30.25 & 20.75 & 3.065 & 1.70 & 20.1 & $\underline{22.25}$ & 8.70 \\
\hline
\end{tabular}

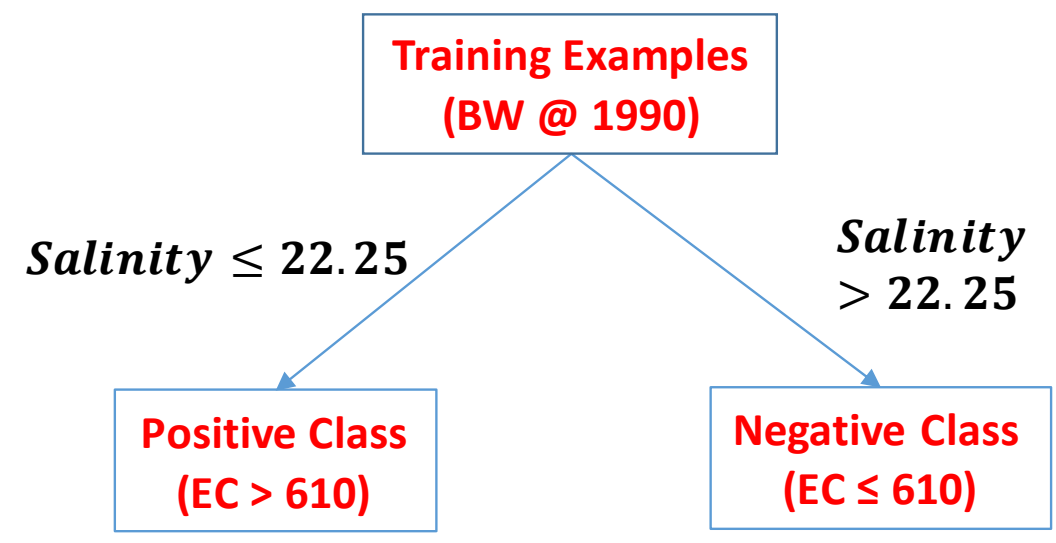

Figure S2: Summary of best split point for each feature (upper table) and the final decision tree (lower figure). In this example, salinity with best split condition of "If salinity $\leq 22.25$ then positive class $(\mathrm{EC}>610)$ " gives the smallest value of min. IG among all features, which will therefore be selected as the final decision stump 